\title{
A Membrane Pool Retrieved via Endocytosis Overshoot at Nerve Terminals: A Study of Its Retrieval Mechanism and Role
}

\author{
Lei Xue, Benjamin D. McNeil, Xin-Sheng Wu, Fujun Luo, Liming He, and Ling-Gang Wu \\ National Institute of Neurological Disorders and Stroke, Bethesda, Maryland 20892
}

Endocytosis overshoot, which retrieves more membrane than vesicles just being exocytosed, occurs at nerve terminals and non-neuronal secretory cells. The mechanism that retrieves the overshoot membrane pool and the role of this pool remain largely unknown. We addressed this issue at the rat calyx of Held nerve terminal with capacitance measurements. We found that every calyx contained an overshoot pool $\sim 1.8$ times the readily releasable pool. Retrieval of this pool required large calcium influx, and was inhibited by blockers of calcium/calmodulin-activated calcineurin and dynamin, suggesting the involvement of calcineurin and dynamin in endocytosis overshoot. Depletion of the overshoot pool slowed down compensatory endocytosis, whereas recovery of the overshoot pool via exocytosis that deposited stranded vesicles to the plasma membrane led to recovery of compensatory endocytosis, suggesting that the overshoot pool enhances endocytosis efficiency. These results suggest that the overshoot pool exists at every nerve terminal, is of limited size arising from vesicles stranded at the plasma membrane, is retrieved via calcium/calmodulin/calcineurin and dynamin signaling pathway, and can enhance endocytosis efficiency. Potential mechanisms for how the endocytosis overshoot pool enhances endocytosis efficiency are discussed.

\section{Introduction}

Endocytosis overshoot, which retrieves more membrane than vesicles just being exocytosed, has been observed in nonneuronal secretory cells and nerve terminals (Thomas et al., 1994; Smith and Neher, 1997; Renden and von Gersdorff, 2007; Wu et al., 2009). However, it is only observed in a fraction of cells, raising a question as to its popularity. Likely because of this reason, the mechanism mediating retrieval of the overshoot membrane pool and the role of this pool are poorly understood. Early works consider calcium as a potential trigger of endocytosis overshoot (Thomas et al., 1994; Smith and Neher, 1997). Recent observation that the overshoot takes place most often after larger calcium influx and is blocked by the calcium buffer EGTA suggests that calcium influx triggers endocytosis overshoot (Wu et al., 2009). A calmodulin blocker inhibits the endocytosis overshoot, suggesting that calmodulin is involved in endocytosis overshoot (Wu et al., 2009). In addition to calcium/calmodulin, little is known about the mechanisms mediating endocytosis overshoot. For example, it remains unclear whether calcineurin, a calcium/calmodulin-activated phosphatase that dephosphorylates many endocytic proteins (Cousin and Robinson, 2001), is

Received Nov. 29, 2011; revised Jan. 6, 2012; accepted Jan. 19, 2012.

Author contributions: L.X., B.D.M., X.-S.W., and L.-G.W. designed research; L.X., F.L., and L.H. performed research; L.X. analyzed data; L.-G.W. wrote the paper.

This work was supported by the National Institute of Neurological Disorders and Stroke Intramural Research Program.

Correspondence should be addressed to Ling-Gang Wu, National Institute of Neurological Disorders and Stroke, 35 Convent Drive, Building 35, Rm. 2B-1012, Bethesda, MD 20892. E-mail: wul@ninds.nih.gov.

DOI:10.1523/JNEUROSCI.5943-11.2012

Copyright $\odot 2012$ the authors $\quad 0270-6474 / 12 / 323398-07 \$ 15.00 / 0$ involved in endocytosis overshoot. It is not known whether dynamin, the fission protein in compensatory endocytosis, is involved in endocytosis overshoot.

What generates the overshoot membrane pool? Block of dynamin-dependent endocytosis led to accumulation of a large amount of fused vesicles at the plasma membrane, which were all retrieved via dynamin-independent endocytosis overshoot, suggesting that overshoot membrane pool originates from vesicles stranded at the plasma membrane (see Wu et al., 2009, their Fig. 8). However, this observation was obtained when GTP hydrolysis was blocked. Whether it applies to control conditions remains unclear.

Endocytosis overshoot could be induced by physiological high-frequency action potential-like stimulation, suggesting that the overshoot pool is to increase the endocytosis capacity and thus to sustain synaptic transmission during high-frequency firing (Renden and von Gersdorff, 2007; Wu et al., 2009). Whether this is the only function of the overshoot pool is unclear.

We addressed the above unclear issues at the calyx nerve terminal, where overshoot can be monitored with capacitance measurements. We determined whether (1) every nerve terminal contains an overshoot membrane pool, (2) calcineurin and dynamin are involved in endocytosis overshoot, (3) endocytosis overshoot retrieves vesicles stranded at the plasma membrane, and (4) the overshoot pool enhances compensatory endocytosis efficiency.

\section{Materials and Methods}

Parasagittal brainstem slices (200 $\mu \mathrm{m}$ thick) containing the medial nucleus of the trapezoid body were prepared from 7- to 10-d-old Wistar rats 
A

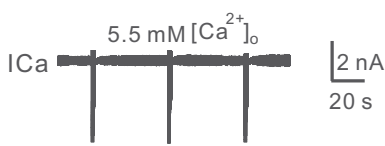

$\mathrm{C}_{\mathrm{m}}-\underbrace{}_{\frac{1 \mathrm{pF}}{20 \mathrm{~s}}}$
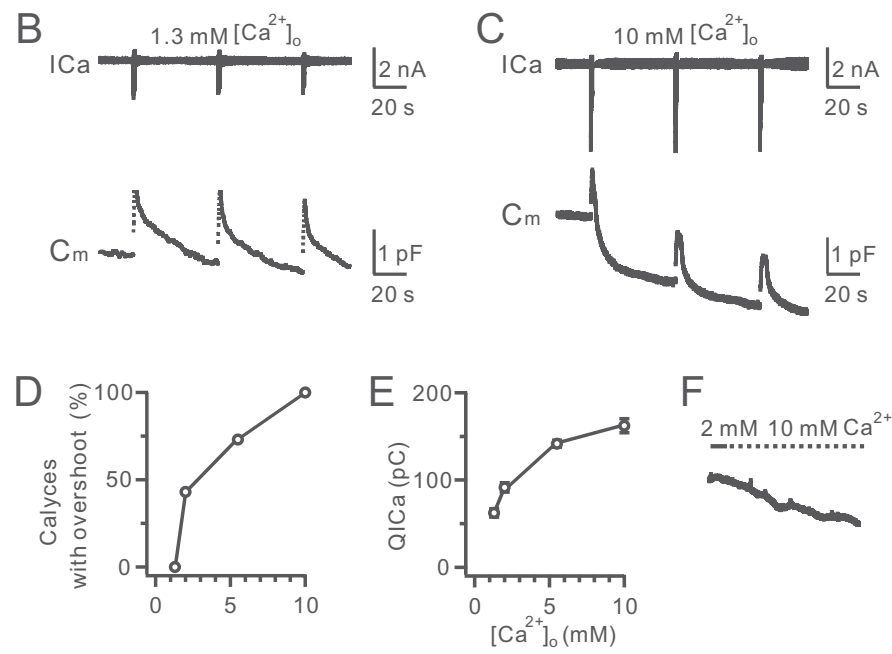

$\mathrm{F}$

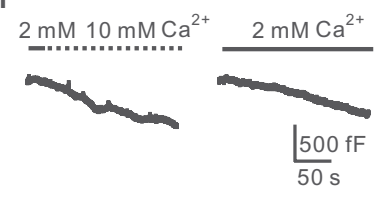

Figure 1. Overshoot pool at every calyx. $A$, Sampled $I_{C_{a}}$ and membrane capacitance $\left(C_{\mathrm{m}}\right)$ induced by 3 depol $I_{50 \mathrm{~ms}} \times 10$ (interval: $50 \mathrm{~s}$ ) at $\left[\mathrm{Ca}^{2+}\right]_{0}=5.5 \mathrm{~mm}$. Overshoot was observed in the left, but not in the right panel (another calyx). B, $\boldsymbol{C}$, Sampled $I_{\mathrm{Ca}}$ and $C_{\mathrm{m}}$ induced by 3 depol $_{50 \mathrm{~ms} \times 10}$ (interval: $50 \mathrm{~s}$ ) at $\left[\mathrm{Ca}^{2+}\right]_{0}=1.3 \mathrm{~mm}(\boldsymbol{B})$ or $10 \mathrm{~mm}$ (C). $\boldsymbol{D}$, The percentage of calyces showing overshoot after 2-3 depol $50 \mathrm{~ms} \times 10$ plotted versus the $\left[\mathrm{Ca}^{2+}\right]_{0}(1.3 \mathrm{~mm}, n=7 ; 2 \mathrm{~mm}, n=14 ; 5.5 \mathrm{~mm}, n=37 ; 10 \mathrm{~mm}, n=15) . \boldsymbol{E}, T_{\text {The }} Q_{\mathrm{I}_{\mathrm{a}}}$ induced by the first $50 \mathrm{~ms}$ depolarization during a depol ${ }_{50 \mathrm{~ms}} \times 10$ plotted versus the $\left[\mathrm{Ca}^{2+}\right]_{0}(1.3 \mathrm{~mm}, n=7 ; 2 \mathrm{~mm}, n=14 ; 5.5 \mathrm{~mm}$, $n=37 ; 10 \mathrm{~mm}, n=15) . \boldsymbol{F}, C_{\mathrm{m}}$ trace at $2 \mathrm{~mm}\left[\mathrm{Ca}^{2+}\right]_{0}$ (right) or when the $\left[\mathrm{Ca}^{2+}\right]_{0}$ changed from 2 to $10 \mathrm{~mm}$ (left).

of either sex using a vibratome. Whole-cell capacitance measurements were made with the EPC-10 amplifier together with the software lock-in amplifier (PULSE, HEKA) that implements Lindau-Neher's technique (Sun and $\mathrm{Wu}, 2001$ ). The sinusoidal stimulus frequency was $1000 \mathrm{~Hz}$, and its peak-to-peak voltage was $\leq 60 \mathrm{mV}$. We isolated $\mathrm{Ca}^{2+}$ currents with a bath solution $\left(\sim 22-24^{\circ} \mathrm{C}\right)$ containing (in mM): $105 \mathrm{NaCl}, 20$ TEA-Cl, $2.5 \mathrm{KCl}, 1 \mathrm{MgCl}_{2}, 2$ or $5.5 \mathrm{CaCl}_{2}, 25 \mathrm{NaHCO}_{3}, 1.25 \mathrm{NaH}_{2} \mathrm{PO}_{4}$, 25 glucose, 0.4 ascorbic acid, 3 myo-inositol, 2 sodium pyruvate, 0.001 tetrodotoxin, 0.1 3,4-diaminopyridine, $\mathrm{pH} 7.4$, when bubbled with $95 \% \mathrm{O}_{2}$ and $5 \% \mathrm{CO}_{2}$. To increase the $\left[\mathrm{Ca}^{2+}\right]_{0}$ to $10 \mathrm{~mm}$, We used a HEPES solution containing (in mM): $105 \mathrm{NaCl}, 20$ TEA-Cl, $2.5 \mathrm{KCl}, 1$ $\mathrm{MgCl}_{2}, 10 \mathrm{CaCl}_{2}, 25$ dextrose, 10 HEPES, 0.4 ascorbic acid, 3 myoinositol, 2 sodium pyruvate, 0.001 TTX. Application of this solution was limited to $\sim 5-6 \mathrm{~min}$ and the solution was bubbled with $95 \% \mathrm{O}_{2}$ and $5 \% \mathrm{CO}_{2}$, in which we did not see any significant rundown of the electrical properties of the calyx. The presynaptic pipette contained (in $\mathrm{mm}$ ): 125 Cs-gluconate, $20 \mathrm{CsCl}, 4 \mathrm{MgATP}, 10 \mathrm{Na}_{2}$ phosphocreatine, 0.3 GTP, 10 HEPES, 0.05 BAPTA, pH 7.2, adjusted with $\mathrm{CsOH}$. The BAPTA concentration $(50 \mu \mathrm{M})$ mimicked the endogenous calcium buffer capacity at calyces (Helmchen et al., 1997).

The statistical test was a $t$ test. Means are presented as \pm SE. The initial

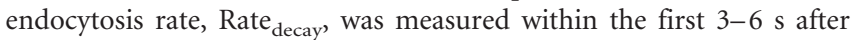
stimulus (Wu et al., 2009). For measuring overshoot, $2-3$ depol $_{50 \mathrm{~ms}} \times 10$ ( 10 pulses of $50 \mathrm{~ms}$ depolarization to $+10 \mathrm{mV}$ at $10 \mathrm{~Hz}$ ) were applied at an interval of 20-50 s. With an interval of $50 \mathrm{~s}$, the overshoot amplitude was calculated as the sum of the overshoot at $40 \mathrm{~s}$ after each depol ${ }_{50 \mathrm{~ms}} \times$ 10. The baseline capacitance drift was corrected based on the linear fit of the baseline within $20 \mathrm{~s}$ before each depol $_{50 \mathrm{~ms}} \times 10$. The resulting overshoot pool size ( $1085 \pm 110 \mathrm{fF}, n=27)$ in calyces showing overshoot at $5.5 \mathrm{~mm}\left[\mathrm{Ca}^{2+}\right]_{0}$ was similar to the accumulated overshoot amplitude $(928 \pm 83 \mathrm{fF}, n=23)$ measured at $20 \mathrm{~s}$ after 2 depol $_{50 \mathrm{~ms} \times 10}$ (interval:
$20 \mathrm{~s})$ at $10 \mathrm{~mm}\left[\mathrm{Ca}^{2+}\right]_{\mathrm{o}}$, in which the small capacitance drift was neglected. Furthermore, the small drift during 2 depol $_{50 \mathrm{~ms}} \times 10$ was likely similar to the small residual overshoot that we did not measure $20 \mathrm{~s}$ later.

\section{Results \\ Every calyx contains an endocytosis overshoot pool}

To determine whether every calyx contains an endocytosis overshoot pool, we induced overshoot with 10 pulses of $50 \mathrm{~ms}$ depolarization (all pulses from -80 to $+10 \mathrm{mV}$ if not mentioned) at $10 \mathrm{~Hz}$ (de$\left.\mathrm{pol}_{50 \mathrm{~ms} \times 10}\right)(\mathrm{Wu}$ et al., 2009) at the extracellular calcium concentration $\left(\left[\mathrm{Ca}^{2+}\right]_{\mathrm{o}}\right)$ of $1.3-10 \mathrm{~mm}$. At $\left[\mathrm{Ca}^{2+}\right]_{\mathrm{o}}=$ $5.5 \mathrm{~mm}$, repeating depol ${ }_{50 \mathrm{~ms}} \times 102$ times (interval: 20-50 s) depleted the overshoot pool (Wu et al., 2009), because the overshoot induced by the third depol $\mathrm{l}_{50 \mathrm{~ms}} \times 10$ was negligible (Fig. 1A). Thus, the overshoot was calculated as the sum induced by the first 2 depol $_{50 \mathrm{~ms}} \times 10$. In 27 of 37 calyces, we observed significant endocytosis overshoot $(>250 \mathrm{fF})$ of $1085 \pm 110 \mathrm{fF}$ ( $n=27$, Fig. $1 A$, left). In the remaining 10 calyces, overshoot was negligible ( $45 \pm 43$ fF, Fig. $1 A$, right)

As the $\left[\mathrm{Ca}^{2+}\right]_{\mathrm{o}}$ increased from 1.3 to 2 , 5.5 , and $10 \mathrm{~mm}$, calyces showing overshoot increased from 0 ( 0 of 7 calyces, Fig. $1 B$ ) to $43 \%$ (6 of 14 calyces), $73 \%$ (27 of 37 calyces, Fig. $1 A$ ), and $100 \%$ (15 of 15 calyces, Fig. $1 C$; data summarized in Fig. $1 D)$. The increase was accompanied by the calcium current $\left(I_{\mathrm{Ca}}\right)$ charge $\left(Q_{\mathrm{I}_{\mathrm{Ca}}}\right)$ increase (Fig. $\left.1 E\right)$. These results suggest that every calyx contained an overshoot membrane pool, which could only be depleted by large calcium influx.

At lower $\left[\mathrm{Ca}^{2+}\right]_{0}$, although we could not induce overshoot at every calyx, the overshoot pool did exist. This was because we applied $10 \mathrm{~mm}$ extracellular calcium after the whole-cell configuration was established (holding at $-80 \mathrm{mV}$ ) at $2 \mathrm{mM}\left[\mathrm{Ca}^{2+}\right]_{\mathrm{o}}$ (Fig. $1 F$, left), which took $\sim 1-2$ min for completion. The switch to $10 \mathrm{~mm}$ calcium did not increase the capacitance (Fig. $1 F$, left). Instead, we observed a baseline capacitance decay $(n=5$, Fig. $1 F$, left) similar to that at $2 \mathrm{~mm}\left[\mathrm{Ca}^{2+}\right]_{\mathrm{o}}(n=4$, Fig. $1 F$, right $)$. The reason for this baseline decay was unclear. Nevertheless, increasing the $\left[\mathrm{Ca}^{2+}\right]_{\mathrm{o}}$ did not deposit stranded vesicles at the plasma membrane for generating the overshoot pool. The endocytosis overshoot pool must exist before the $\left[\mathrm{Ca}^{2+}\right]_{0}$ was increased to $10 \mathrm{~mm}$.

\section{Blockers of calcineurin and dynamin inhibit endocytosis overshoot}

To study the role of calcineurin, we included in the pipette either the calcineurin autoinhibitory peptide $\left(\mathrm{CaN}_{457-482}, 150 \mu \mathrm{M}\right)$ or cyclosporine A (CsA, 20-50 $\mu \mathrm{M}$ ) (Oliveria et al., 2007). These two blockers are specific at calyces because they inhibit rapid and slow compensatory endocytosis as knocking out calcineurin $\mathrm{A}_{\alpha}$ subunit at calyces (Sun et al., 2010). We induced endocytosis overshoot by 3 depol $_{50 \mathrm{~ms} \times 10}$ applied every $50 \mathrm{~s}$ at $\left[\mathrm{Ca}^{2+}\right]_{\mathrm{o}}=5.5 \mathrm{mM}$. In the presence of $\mathrm{CaN}_{457-482}$, endocytosis overshoot was blocked 
A

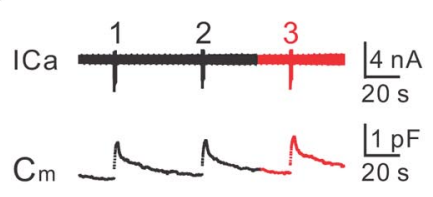

B

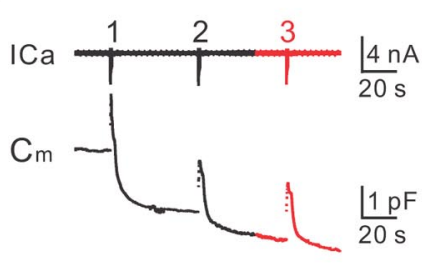

C

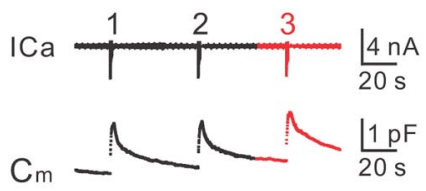

$\mathrm{CaN}_{\mathrm{P}}$

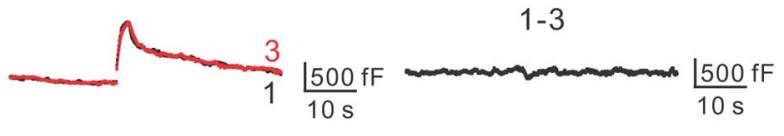

$\mathrm{SCaN}_{\mathrm{P}}$

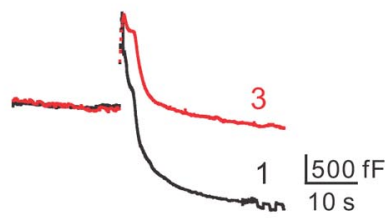

1-3

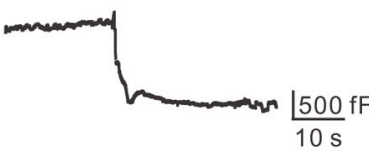

CsA

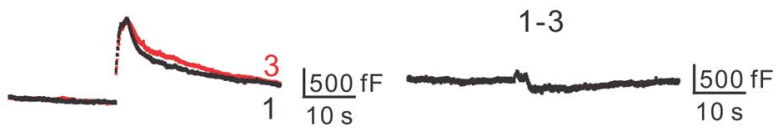

D

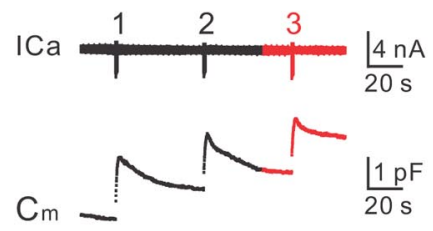

Dynasore

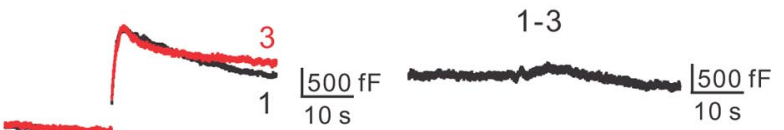

E

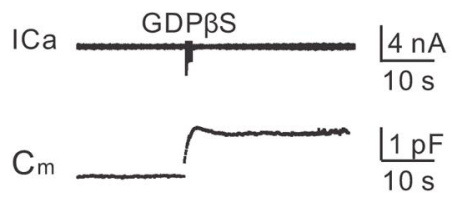

F

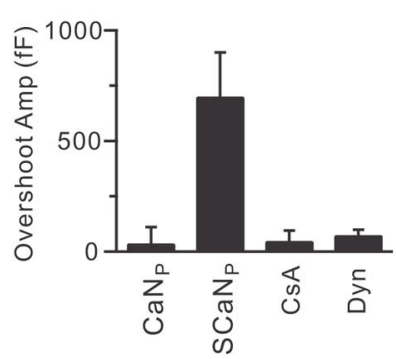

Figure 2. Calcineurin and dynamin blockers inhibit endocytosis overshoot. $A-D$, Sampled $I_{C_{a}}$ and $C_{\mathrm{m}}$ induced by 3 depol ${ }_{50 \mathrm{~ms}} \times 10$ applied every 50 s in the presence of $\mathrm{CaN}_{457-482}\left(\mathrm{CaN}_{\mathrm{p}}, 150 \mu \mathrm{M}\right.$ in pipette, $\boldsymbol{A})$, scrambled $\mathrm{CaN}_{457-482}\left(\mathrm{SCaN}_{\mathrm{p}}, 150 \mu \mathrm{m}, \boldsymbol{B}\right), \mathrm{CSA}(20 \mu \mathrm{m}$ in pipette, $\boldsymbol{C})$, or dynasore (100 $\mu \mathrm{m}$ in pipette, $\left.\boldsymbol{D}\right)$. $\boldsymbol{C}_{\mathrm{m}}$ change induced by the third and the first depol ${ }_{50} \mathrm{~ms} \times 10$ was superimposed (middle). Subtraction of the third $C_{\mathrm{m}}$ response from the first one yields a trace reflecting endocytosis overshoot only (right). $\boldsymbol{E}$, Sampled $I_{\mathrm{Ca}_{2}}$ and $C_{\mathrm{m}}$ induced by a depol ${ }_{50} \mathrm{~ms} \times 10$ in the presence of $\operatorname{GDP} \beta S\left(0.3 \mathrm{~mm}\right.$ in pipette). $\boldsymbol{F}$, The overshoot amplitude induced by the first depol ${ }_{50 \mathrm{~ms}} \times 10$, calculated as the difference between the first and the third $\boldsymbol{C}_{\mathrm{m}}$ response $(\boldsymbol{A}-\boldsymbol{D})$, plotted in the presence of $\mathrm{CaN}_{\mathrm{p}}(n=7), \mathrm{SCaN}_{\mathrm{p}}(n=7), \mathrm{CsA}(n=9)$ or dynasore (Dyn, $\left.n=15\right)$.

in 7 of 7 calyces. In contrast, in the presence of scrambled $\mathrm{CaN}_{457-482}$ $(150 \mu \mathrm{M})$, endocytosis overshoot was observed in 6 of 7 calyces with an amplitude $\left(1050 \pm 302 \mathrm{fF}, n=7\right.$, Fig. $2 B, \chi^{2}$ test, $p<$ 0.01 ) similar to that of the control (Fig. 1). Like $\mathrm{CaN}_{457-482}$, CsA $(20-50 \mu \mathrm{M})$ abolished endocytosis overshoot in 9 of 9 calyces (Fig. 2C, $\chi^{2}$ test, $p<0.01$ ).

In the presence of $\mathrm{CaN}_{457-482}$ or CsA, the capacitance decay was slowed down, but not abolished (Fig. $2 A, C$ ). One may argue that the capacitance decay reflected endocytosis overshoot, whereas the compensatory endocytosis was abolished. This scenario predicts that endocytosis overshoot in the presence of calcineurin blockers should be similar to control, that is, endocytosis overshoot should be induced by the first 2 depol $_{50 \mathrm{~ms} \times 10}$, but not the third one. In other words, the capacitance decay induced by the first depol $_{50 \mathrm{~ms}} \times{ }_{10}$ should be much larger than that by the third depol ${ }_{50 \mathrm{~ms}} \times 10$. This prediction was incorrect, as quantified below (Fig. $2 A, C$ ).

In control (with scrambled $\mathrm{CaN}_{457-482}$ ), the first $\operatorname{depol}_{50 \mathrm{~ms}} \times 10$ generated an overshoot of $785 \pm 236 \mathrm{fF}(n=7$, included calyces with or without overshoot), whereas the third depol $_{50 \mathrm{~ms}} \times 10$ induced only compensatory endocytosis (Fig. $2 B$; see also Fig. $1 A)$. By subtracting the capacitance decay induced by the third de$\mathrm{pol}_{50 \mathrm{~ms} \times 10}$ from that by the first $\operatorname{depol}_{50 \mathrm{~ms} \times 10}$, the difference reflected purely the endocytosis overshoot with an amplitude $(702 \pm 198 \mathrm{fF}, n=7$, Fig. $2 B)$ similar to that $(785 \pm 236 \mathrm{fF}, n=$ 7) measured directly from the first capacitance response. However, same subtraction in the presence of $\mathrm{CaN}_{457-482}$ or CsA revealed negligible overshoot (Fig. $2 \mathrm{~F}, \mathrm{CaN}_{457-482}: 38 \pm 73 \mathrm{fF}$, $n=7$; CsA: $48 \pm 47 \mathrm{fF}, n=9$; summarized). Thus, $\mathrm{CaN}_{457-482}$ and CsA abolished overshoot. 
A
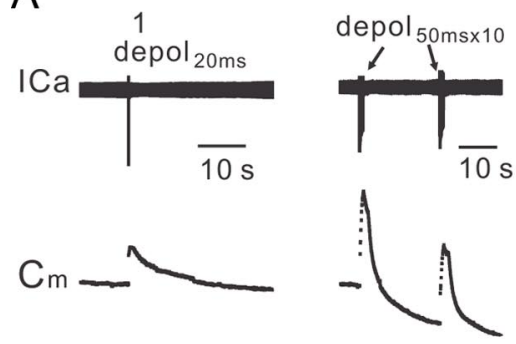

C

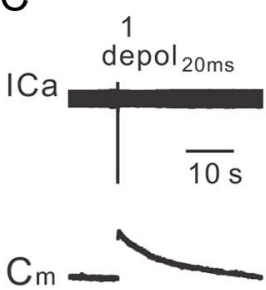

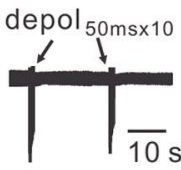

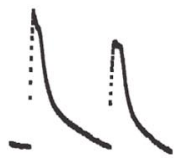

\section{2}
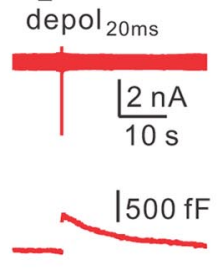

$1+2$

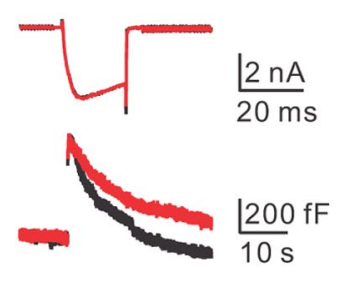

2

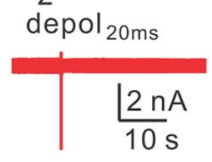

$-1500 \mathrm{fF}$

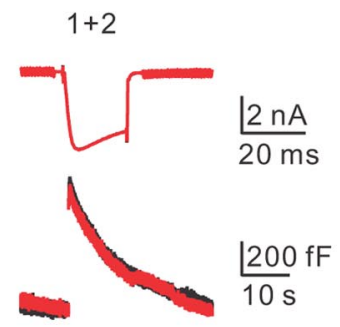

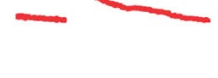

B

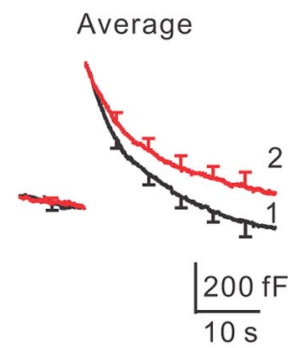

D

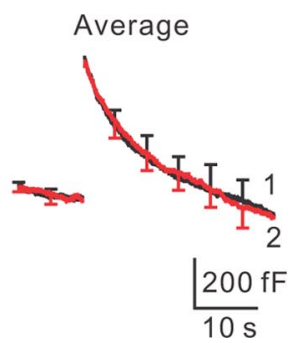

Figure 3. Overshoot pool depletion slows down endocytosis. $A, S$ Sampled $I_{C_{a}}$ and $C_{\mathrm{m}}$ from a calyx stimulated with a depol $\mathrm{I}_{20 \mathrm{~ms}}$ (first, black) and $40 \mathrm{~s}$ rest (left), 2 depol ${ }_{50 \mathrm{~ms}} \times 10$ (interval: $20 \mathrm{~s}$, middle left), and another depol $\mathrm{I}_{20 \mathrm{~ms}} 40$ slater (second, middle right, red). Traces induced by first and second depol $\mathrm{I}_{20 \mathrm{~ms}}$ are superimposed (right). $\boldsymbol{B}$, The mean $\boldsymbol{C}_{\mathrm{m}}$ induced by first and second depo $\mathrm{I}_{20 \mathrm{~ms}}$ ( ${ }$ ame protocol as $\boldsymbol{A}$ ) from 30 calyces showing significant overshoot ( $>250 \mathrm{fF}$, data expressed as $+\mathrm{SE}$ or $-\mathrm{SE}$ every $5 \mathrm{~S}$ ). $\boldsymbol{C}, \boldsymbol{D}$, Similar to $\boldsymbol{A}$ and $\boldsymbol{B}$, except from 9 calyces showing no significant overshoot.

Although unlikely, we could not exclude the possibility that overshoot was only partially inhibited by $\mathrm{CaN}_{457-482}$ and CsA so that the first and the third depol $_{50 \mathrm{~ms}} \times{ }_{10}$ induced similar, yet smaller endocytosis overshoot. However, even with this scenario, our conclusion that $\mathrm{CaN}_{457-482}$ and CsA inhibited overshoot holds.

To determine whether the GTPase dynamin is involved in endocytosis overshoot, we included in the pipette either dynasore $(100 \mu \mathrm{M}$, Fig. $2 D)$ or GDP $\beta S(0.3 \mathrm{~mm}$, Fig. $2 E)$ to inhibit the GTPase activity of dynamin (Macia et al., 2006). Dynasore is specific to dynamin at calyces, because it inhibits endocytosis as dynamin I knock-out at calyces (Lou et al., 2008; Xu et al., 2008). In the presence of dynasore, $3 \operatorname{depol}_{50 \mathrm{~ms} \times 10}$ applied at $50 \mathrm{~s}$ interval slowed down the capacitance decay and induced no overshoot $(n=15)$. Subtraction of the third response from the first response revealed that the first $\operatorname{depol}_{50 \mathrm{~ms}} \times 1{ }_{10}$ induce negligible overshoot (Fig. 2D,F). GDP $\beta$ S blocked overshoot, because the capacitance decay after a depol ${ }_{50 \mathrm{~ms} \times 10}$ was nearly abolished $(n=$ 5 , Fig. $2 E$ ). These results suggest the involvement of dynamin in endocytosis overshoot.

\section{Depletion of the endocytosis overshoot pool slows down endocytosis}

To determine whether the overshoot pool enhances endocytosis efficiency, we applied a stimulation protocol composed of a $20 \mathrm{~ms}$ depolarization $\left(\operatorname{depol}_{20 \mathrm{~ms}}\right)$ and $40 \mathrm{~s}$ rest for measuring the endocytosis rate, 2 depol $_{50 \mathrm{~ms}} \times 10$ (interval: $20 \mathrm{~s}$ ) to deplete the overshoot pool, and another depol $_{20 \mathrm{~ms}}$ (second) applied at $40 \mathrm{~s}$ after the second depol ${ }_{50 \mathrm{~ms} \times 10}$ for measuring the endocytosis rate (Fig. $3 A)$. The $\left[\mathrm{Ca}^{2+}\right]_{\mathrm{o}}$ was $5.5 \mathrm{~mm}$. The first $\mathrm{depol}_{20 \mathrm{~ms}}$, which depletes the readily releasable vesicle pool (Sun and $\mathrm{Wu}, 2001$ ), induced a capacitance jump $\left(\Delta C_{\mathrm{m}}\right)$ of $541 \pm 20 \mathrm{fF}(n=39$, e.g., Fig. $3 A$, left). The jump was followed by a monoexponential decay with a $\tau$ of $10.9 \pm 0.7 \mathrm{~s}$ in 35 calyces, and by a biexponential decay in 4 calyces with $\tau$ of $0.4 \pm 0.2 \mathrm{~s}(25 \pm 4 \%)$ and $8.9 \pm 1.6 \mathrm{~s}$, respectively. The initial capacitance decay, which reflects the initial endocytosis rate (Rate $_{\text {endo }}$, measured within 3-6 s after stimula- tion), was $76 \pm 8 \mathrm{fF} / \mathrm{s}(n=39$, Fig. $3 A$, left $)$. Subsequent 2 depol $_{50 \mathrm{~ms}} \times 10$ depleted the overshoot pool in 30 of 39 calyces with an overshoot amplitude of $1018 \pm 104 \mathrm{fF}(n=30$, Fig. $3 A)$. The second depol $_{20 \mathrm{~ms}}$, induced a $\Delta C_{\mathrm{m}}(528 \pm 24 \mathrm{fF}, n=30)$ similar to that by the first depol $20 \mathrm{~ms}$, but a Rate endo $(53 \pm 5 \mathrm{fF} / \mathrm{s}, n=30, p<0.01)$ significantly slower than that by the first $\operatorname{depol}_{20 \mathrm{~ms}}$ (Fig. $3 A$, see $B$ for the average). The $\tau$ was difficult to quantify because of the slow endocytosis time course, which sometimes did not return to the baseline (Fig. $3 A, B)$. The $Q_{\mathrm{I}_{\mathrm{Ca}}}$ induced by the first $(66.2 \pm 2.4$ $\mathrm{pC}, n=30)$ and the second $\operatorname{depol}_{20 \mathrm{~ms}}(63.6 \pm 2.3 \mathrm{pC}, n=30)$ were similar $(p>0.9$, Fig. $3 A)$.

In 9 of 39 calyces, repeated depol $50 \mathrm{~ms} \times 10$ induced negligible overshoot $(28 \pm 16 \mathrm{fF}, n=9$, Fig. $3 C, D)$. The $Q_{\mathrm{I}_{\mathrm{Ca}}}, \Delta C_{\mathrm{m}}$, endo-

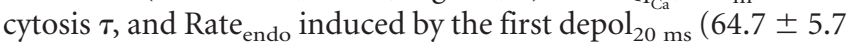
$\mathrm{pC}, 512 \pm 36 \mathrm{fF}, 11.4 \pm 0.9 \mathrm{~s}, 69 \pm 10 \mathrm{fF} / \mathrm{s}, n=9)$ were similar to those induced by the second depol ${ }_{20 \mathrm{~ms}}(62.9 \pm 5.4 \mathrm{pC}, 501 \pm 28$ $\mathrm{fF}, 11.9 \pm 1.2 \mathrm{~s}, 67 \pm 9 \mathrm{fF} / \mathrm{s}, n=9 ; p>0.5$, Fig. $3 C, D)$. These results suggest that overshoot pool depletion slows down the Rate $_{\text {endo }}$.

\section{Recovery of the overshoot pool and endocytosis efficiency}

To determine whether the overshoot pool and the Rate endo $_{\text {could }}$ recover, we applied a stimulation protocol composed of (1) first depol $_{20 \mathrm{~ms}}$ and a $35 \mathrm{~s}$ rest, (2) 2 depol $_{50 \mathrm{~ms} \times 10}$ (interval: $20 \mathrm{~s}$ ) and a $45 \mathrm{~s}$ rest, (3) either $90 \mathrm{~s}$ rest (Fig. $4 \mathrm{~A}$, control protocol) or 6 pulses of 20 $\mathrm{ms}$ depolarization from -80 to $-5 \mathrm{mV}$ at $10 \mathrm{~s}$ interpulse interval and a $40 \mathrm{~s}$ rest (Fig. $4 B$, test protocol), (4) second depol $20 \mathrm{~ms}$ and $35 \mathrm{~s}$ rest, and (5) 2 depol $_{50 \mathrm{~ms} \times 10}$ (interval: $20 \mathrm{~s}$ ). The $\left[\mathrm{Ca}^{2+}\right]_{\mathrm{o}}$ was $10 \mathrm{~mm}$. The overshoot induced by the first 2 depol $_{50 \mathrm{~ms}} \times 10$ was $928 \pm 83 \mathrm{fF}$ $(n=23)$, which was $1.8 \pm 0.2(n=23)$ times the readily releasable pool size, the $\Delta C_{\mathrm{m}}$ induced by a depol $20 \mathrm{~ms}$ (Fig. $4 A$ ).

For the control protocol (Fig. $4 A$, without 6 depolarizing pulses), Rate endo $(43 \pm 4 \mathrm{fF} / \mathrm{s}, n=7)$ induced by the second de$\mathrm{pol}_{20 \mathrm{~ms}}$ was significantly smaller than the first one $(62 \pm 5 \mathrm{fF} / \mathrm{s}$, $n=7, p<0.01$, Fig. $4 A$ ), consistent with results shown in Figure 3. The overshoot induced by the second train of $2 \operatorname{depol}_{50 \mathrm{~ms}} \times 10$ 
A
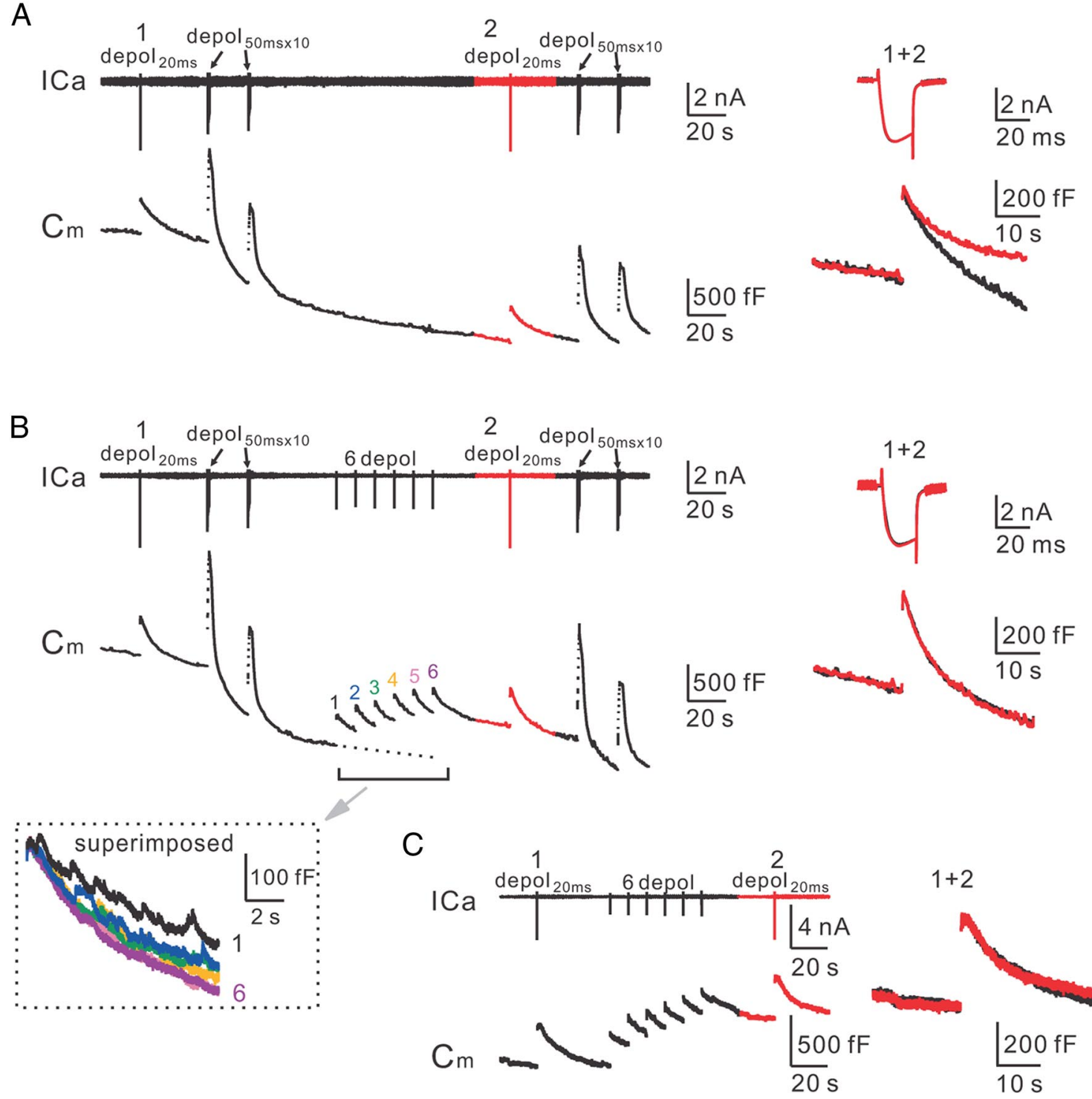

Figure 4. Recovery of the overshoot pool and endocytosis rate. $\boldsymbol{A}, \boldsymbol{B}$, Left, Mean $I_{\mathrm{Ca}}$ and $C_{\mathrm{m}}$ stimulated with a control $(\boldsymbol{A}, n=7)$ or test $(\boldsymbol{B}, n=16)$ protocol described in Results. The dotted line $(\boldsymbol{B})$ is an extrapolation of the capacitance drift. The inset in $\boldsymbol{B}$ shows the capacitance decay after 6 depolarizing pulses (traces are colored-coded and superimposed). The decay is faster as the pulse number increases. Right, $I_{\mathrm{Ca}}$ and $C_{\mathrm{m}}$ induced by first (black) and second (red) depol ${ }_{20 \mathrm{~ms}}$ are superimposed. C, Sampled $I_{\mathrm{Ca}}$ and $C_{\mathrm{m}}$ induced by a depol $I_{20 \mathrm{~ms}}$ (first), 6 depolarizing pulses (interval: $10 \mathrm{~s}$ ) and another depo $\mathrm{I}_{20 \mathrm{~ms}}$ (second). Responses to first and second depo $\mathrm{I}_{20 \mathrm{~ms}}$ are also superimposed (right).

was only $8 \pm 5 \%(n=7)$ of that induced by the first train (Fig. $4 A$ ), indicating negligible overshoot recovery.

For the test protocol (Fig. $4 B$, with 6 depolarizing pulses), the Rate $_{\text {endo }}$ induced by the second $\operatorname{depol}_{20 \mathrm{~ms}}(75 \pm 6 \mathrm{fF} / \mathrm{s}, n=16)$ was similar to that induced by the first $\operatorname{depol}_{20 \mathrm{~ms}}(78 \pm 7 \mathrm{fF} / \mathrm{s}, n=$ $16, p>0.9)$, and the overshoot induced by the second train of 2 depol $_{50 \mathrm{~ms}} \times 10$ was $50 \pm 6 \%(n=16)$ of that induced by the first train (Fig. $4 B$ ). The $\Delta C_{\mathrm{m}}$, the endocytosis time constant(s) and $Q_{\mathrm{I}_{\mathrm{C}}}$ induced by the second depol $20 \mathrm{~ms}$ were similar to those induced by the first $\operatorname{depol}_{20 \mathrm{~ms}}(n=16, p>0.9$; Fig. $4 B)$. Thus, after overshoot pool depletion, vesicle fusion during 6 depolarizing pulses (Fig. $4 \mathrm{~B}$ ) led to partial overshoot pool recovery and the Rate $_{\text {endo }}$ recovery, suggesting that overshoot pool recovery causes the Rate endo $_{\text {recovery. }}$

Partial overshoot recovery predicted that 6 depolarizing pulses generated stranded vesicles at the plasma membrane. As measured $125 \mathrm{~s}$ after the onset of 6 depolarizing pulses, the capacitance increased by $71 \pm 41 \mathrm{fF}(n=16$, Fig. $4 B$, dotted line). In contrast, the capacitance decreased by $443 \pm 54 \mathrm{fF}(n=7)$ at the same time frame, but without 6 depolarizing pulses (Fig. $4 A$, dotted line). Thus, 6 depolarizing pulses generated $\sim 514 \mathrm{fF}$ of stranded vesicles at the plasma membrane, similar to the recovered overshoot amplitude $(460 \pm 23 \mathrm{fF}, n=16)$. The capacitance decrease in the absence of depolarization (Fig. 4A) was due to baseline drift, because a similar drift $(568 \pm 101 \mathrm{fF}, n=5)$ was observed during the same time frame (125 s) of whole-cell recordings at $10 \mathrm{~mm}\left[\mathrm{Ca}^{2+}\right]_{\mathrm{o}}$ (Fig. $1 F$, left). In addition, residual endocytosis overshoot, which was not completed at $20 \mathrm{~s}$ after 2 depol $_{50 \mathrm{~ms} \times 10}$, might contribute to the drift to a minor extent.

During 6 depolarizing pulses, endocytosis rate after each pulse increased gradually (Fig. $4 \mathrm{~B}$, inset). This is consistent with the increase of stranded vesicles that may enhance endocytosis, although it might be also due to accumulation of fused vesicles that recruit more endocytic sites for retrieval (Balaji et al., 2008).

While recovery of the overshoot and Rate decay $_{\text {after }} 6$ depolarizing pulses (Fig. 4) suggests a casual relation between the over- 
shoot pool and Rate $_{\text {decay, }}$ an alternative explanation is that residual calcium generated by 6 depolarizing pulses facilitates

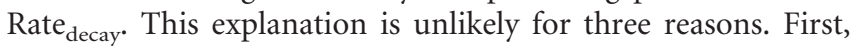
residual calcium generated by each $20 \mathrm{~ms}$ depolarization lasted for only $\sim 1 \mathrm{~s}$ ( $n=5$, our unpublished data; see also $\mathrm{Wu}$ and Borst, 1999). There should be no residual calcium at $40 \mathrm{~s}$ after 6 depolarizing pulses, at which time we measured Rate decay. $_{\text {Sec- }}$ ond, despite using stimuli (e.g., 10 pulses of $20 \mathrm{~ms}$ depolarization at $10 \mathrm{~Hz}$ ) much stronger than 6 depolarizing pulses, the Rate decay measured $40 \mathrm{~s}$ later did not increase (Xu et al., 2008; Wu et al., 2009), suggesting that residual calcium 40 s later does not facilitate endocytosis. Third, without preceding $\operatorname{depol}_{50 \mathrm{~ms}} \times 10$, the Rate $_{\text {decay }}$ induced by a depol $20 \mathrm{~ms} 40 \mathrm{~s}$ before and after the 6 depolarizing pulses did not change significantly (Fig. $4 C, n=5, p>$ 0.8 ), indicating that 6 depolarizing pulses alone could not increase Rate decay $_{\text {. }}$

Could the overshoot pool recovery (Fig. $4 B$ ) be due to residual calcium rather than stranded vesicles produced by 6 depolarizing pulses? This possibility is unlikely for three reasons. First, when GDP $\beta$ S blocks dynamin-dependent endocytosis (Xu et al., 2008), stimulation $\left(11\right.$ depol $_{20 \mathrm{~ms}}$ at $\left.0.1 \mathrm{~Hz}\right)$ induced $3.2 \pm 0.6 \mathrm{pF}(n=5)$ of stranded vesicle membrane at the plasma membrane $(\mathrm{Wu}$ et al., 2009). Subsequent strong stimulation induced dynaminindependent endocytosis and an endocytosis overshoot $(2.5 \pm$ $0.6 \mathrm{pF}, n=5$ ) similar to the accumulated stranded vesicles, indicating that the overshoot is from stranded vesicles ( $\mathrm{Wu}$ et al., 2009). Consistently, stranded vesicles produced by 6 depolarizing pulses $(\sim 514 \mathrm{fF})$ were similar to the recovered overshoot size $(460 \pm 23 \mathrm{fF}, n=16$, Fig. $4 B$ ). Second, regardless of the preceding stimuli, as long as they induced compensatory endocytosis, subsequent $2-3$ depol $_{50 \mathrm{~ms} \times 10}$ did not induce a larger overshoot than those without the preceding stimuli, suggesting that residual calcium alone could not increase overshoot. Third, when the interval of 6 depolarizing pulses was increased to $20 \mathrm{~s}$, the overshoot pool recovery was similar to that with an interval of $10 \mathrm{~s}(n=6$, data not shown).

\section{Discussion}

Our results can be summarized in four aspects. First, previous reports of endocytosis overshoot from only a fraction of cells question whether overshoot is a widespread phenomenon. We found that every nerve terminal contained an overshoot pool $\sim 1.8$ times the readily releasable pool, retrieval of which required large calcium influx (Fig. 1). As calcium influx increased, the fraction of calyces showing overshoot increased (Fig. 1), explaining why overshoot is usually reported from a fraction of cells. Thus, endocytosis overshoot is a widespread phenomenon at nerve terminals. As the $\left[\mathrm{Ca}^{2+}\right]_{\mathrm{o}}$ approached the physiological level of 1.3-2 mM, overshoot was more difficult to induce (Fig. 1), suggesting that the overshoot pool is preserved in many physiological conditions, which may ensure its role in enhancing the endocytosis efficiency.

Second, although endocytosis overshoot retrieves vesicles stranded at the plasma membrane when dynamin-dependent endocytosis is blocked (Wu et al., 2009), it was unclear whether this finding applies to control conditions. The present work showed that in control, fused, but stranded vesicles replenished the overshoot pool after the pool was depleted (Fig. 4). Together, these results suggest stranded vesicles as the source for endocytosis overshoot. Stranded vesicles may be generated in resting or low calcium conditions, in which exocytosis is followed by extremely slow or no endocytosis (von Gersdorff and Matthews, 1994; Hosoi et al., 2009; Wu et al., 2009).
Third, except that calcium/calmodulin was recently found to be involved in endocytosis overshoot (Wu et al., 2009), the mechanism mediating endocytosis overshoot was unclear. We found that calcineurin and dynamin blockers inhibited overshoot. The blockers were specific, because their effects on compensatory endocytosis were confirmed with knock-out of calcineurin and dynamin (Lou et al., 2008; Xu et al., 2008; Sun et al., 2010). Thus, we found two critical molecules, calcineurin and dynamin, in mediating endocytosis overshoot. We suggest that calcium binding with calmodulin activates calcineurin, which dephosphorylates endocytosis proteins, including dynamin (Cousin and Robinson, 2001), leading to the trigger of endocytosis overshoot. Since calcium/calmodulin/calcineurin and dynamin are also involved in rapid and slow compensatory endocytosis at p7-10 calyces examined here (Yamashita et al., 2005, 2010; Xu et al., 2008; Sun et al., 2010), endocytosis overshoot and compensatory endocytosis are mediated by similar mechanisms, except that endocytosis overshoot requires larger calcium influx.

The slow calcium buffer EGTA blocks endocytosis overshoot, but only slows down compensatory endocytosis, whereas the fast calcium buffer BAPTA blocks both overshoot and compensatory endocytosis (Wu et al., 2009). These findings, together with the observed increase of the overshoot frequency at higher calcium influx (Fig. 1), suggest that endocytosis overshoot may be mediated at a place with a mean distance farther from calcium channels. However, a fraction of the overshoot pool membrane might be still near calcium channels to compete with newly exocytosed vesicles for retrieval (more discussion later).

We have previously shown that rapid and slow endocytosis are dynamin dependent, but become dynamin independent after block of dynamin with GDP $\beta$ S or GTP $\gamma S$ when a large number of fused vesicles are accumulated at the plasma membrane (Xu et al., 2008). Similarly, endocytosis overshoot is dynamin dependent as shown here (Fig. 2), but becomes GTP independent and thus dynamin independent after block of dynamin with GDP $\beta S$ when many fused vesicles are accumulated at the plasma membrane (Wu et al., 2009). Thus, similar to rapid and slow endocytosis (Xu et al., 2008), endocytosis overshoot could also be dynamin dependent and independent. Different vesicle pools that use dynamin or an unidentified fission molecule for fission might provide an explanation for the existence of dynamin-dependent and -independent forms of rapid, slow and overshoot endocytosis.

Fourth, overshoot pool depletion slowed down slow endocytosis (Figs. 3,4), the dominant form of clathrin-dependent endocytosis at synapses (Granseth et al., 2006; Kim and Ryan, 2009). After overshoot pool depletion, recovery of the overshoot pool by depositing stranded vesicles at the plasma membrane led to re-

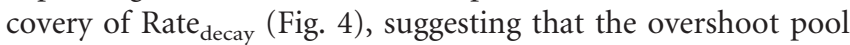
enhances endocytosis efficiency.

We proposed that stranded vesicles at the plasma membrane compete with newly exocytosed vesicles for retrieval. It is possible that stranded vesicle membrane forms a preassembled vesicular structure, which may be advantageous for retrieval by saving many steps involved in retrieving newly exocytosed vesicles, such as sorting and transportation of membrane and proteins to endocytic sites, and membrane invagination. While it seems more likely for stranded vesicles to be retrieved, newly exocytosed vesicles can also be retrieved. Consistent with this possibility, endocytosis induced by depol 20 ms was slower but not abolished after overshoot pool depletion (Figs. 3,4). Endocytosis induced by a depol $_{50 \mathrm{~ms} \times 10}$ also slowed down after overshoot pool depletion, but was still much faster than that induced by $\operatorname{depol}_{20 \mathrm{~ms}}$ (Figs. 
$3,4)$, in agreement with regulation of endocytosis by calcium (Sankaranarayanan and Ryan, 2001; Hosoi et al., 2009; Wu et al., 2009).

In summary, using capacitance measurements to monitor vesicle's lipid membrane turnover, the present work suggests that the overshoot pool exists at every nerve terminal, is of limited size arising from vesicles stranded at the plasma membrane, is retrieved via calcium/calmodulin/calcineurin and dynamin signaling pathway, and can enhance endocytosis efficiency. These findings complement recent observation of a plasma membrane pool of vesicle proteins, including synaptotagmin, VAMP2, synaptophysin, and the vesicular glutamate transporter vGlu1, which may be preferentially retrieved (but see Willig et al., 2006; Fernández-Alfonso et al., 2006; Wienisch and Klingauf, 2006; Hua et al., 2011).

Endocytosis overshoot could be induced by physiological high-frequency action potential-like stimulation, suggesting that the overshoot pool increases the endocytosis capacity during high-frequency firing (Renden and von Gersdorff, 2007; Wu et al., 2009). The present work suggests that the overshoot pool can also enhance the efficiency of compensatory endocytosis. These functions may help nerve terminals with a limited number of recycling vesicles to maintain synaptic transmission during repetitive firing. Although these functions were found in calyces, their significance is likely to apply beyond calyces, because endocytosis overshoot was found at other nerve terminals and endocrine cells (Thomas et al., 1994; Hsu and Jackson, 1996; Smith and Neher, 1997).

\section{References}

Balaji J, Armbruster M, Ryan TA (2008) Calcium control of endocytic capacity at a CNS synapse. J Neurosci 28:6742-6749.

Cousin MA, Robinson PJ (2001) The dephosphins: dephosphorylation by calcineurin triggers synaptic vesicle endocytosis. Trends Neurosci 24:659-665.

Fernández-Alfonso T, Kwan R, Ryan TA (2006) Synaptic vesicles interchange their membrane proteins with a large surface reservoir during recycling. Neuron 51:179-186.

Granseth B, Odermatt B, Royle SJ, Lagnado L (2006) Clathrin-mediated endocytosis is the dominant mechanism of vesicle retrieval at hippocampal synapses. Neuron 51:773-786.

Helmchen F, Borst JG, Sakmann B (1997) Calcium dynamics associated with a single action potential in a CNS presynaptic terminal. Biophys J 72:1458-1471.

Hosoi N, Holt M, Sakaba T (2009) Calcium dependence of exo- and endocytotic coupling at a glutamatergic synapse. Neuron 63:216-229.

Hsu SF, Jackson MB (1996) Rapid exocytosis and endocytosis in nerve terminals of the rat posterior pituitary. J Physiol 492:539-553.
Hua Y, Sinha R, Thiel CS, Schmidt R, Hüve J, Martens H, Hell SW, Egner A, Klingauf J (2011) A readily retrievable pool of synaptic vesicles. Nat Neurosci 14:833-839.

Kim SH, Ryan TA (2009) Synaptic vesicle recycling at CNS synapses without AP-2. J Neurosci 29:3865-3874.

Lou X, Paradise S, Ferguson SM, De Camilli P (2008) Selective saturation of slow endocytosis at a giant glutamatergic central synapse lacking dynamin 1. Proc Natl Acad Sci U S A 105:17555-17560.

Macia E, Ehrlich M, Massol R, Boucrot E, Brunner C, Kirchhausen T (2006) Dynasore, a cell-permeable inhibitor of dynamin. Dev Cell 10:839-850.

Oliveria SF, Dell'Acqua ML, Sather WA (2007) AKAP79/150 anchoring of calcineurin controls neuronal L-type $\mathrm{Ca}^{2+}$ channel activity and nuclear signaling. Neuron 55:261-275.

Renden R, von Gersdorff H (2007) Synaptic vesicle endocytosis at a CNS nerve terminal: faster kinetics at physiological temperatures and increased endocytotic capacity during maturation. J Neurophysiol 98:3349-3359.

Sankaranarayanan S, Ryan TA (2001) Calcium accelerates endocytosis of vSNAREs at hippocampal synapses. Nat Neurosci 4:129-136.

Smith C, Neher E (1997) Multiple forms of endocytosis in bovine adrenal chromaffin cells. J Cell Biol 139:885-894.

Sun JY, Wu LG (2001) Fast kinetics of exocytosis revealed by simultaneous measurements of presynaptic capacitance and postsynatpic currents at a central synapse. Neuron 30:171-182.

Sun T, Wu XS, Xu J, McNeil BD, Pang ZP, Yang W, Bai L, Qadri S, Molkentin JD, Yue DT, Wu LG (2010) The role of calcium/calmodulin-activated calcineurin in rapid and slow endocytosis at central synapses. J Neurosci 30:11838-11847.

Thomas P, Lee AK, Wong JG, Almers W (1994) A triggered mechanism retrieves membrane in seconds after $\mathrm{Ca}^{2+}$-stimulated exocytosis in single pituitary cells. J Cell Biol 124:667-675.

von Gersdorff H, Matthews G (1994) Inhibition of endocytosis by elevated internal calcium in a synaptic terminal. Nature 370:652-655.

Wienisch M, Klingauf J (2006) Vesicular proteins exocytosed and subsequently retrieved by compensatory endocytosis are nonidentical. Nat Neurosci 9:1019-1027.

Willig KI, Rizzoli SO, Westphal V, Jahn R, Hell SW (2006) STED microscopy reveals that synaptotagmin remains clustered after synaptic vesicle exocytosis. Nature 440:935-939.

Wu LG, Borst JG (1999) The reduced release probability of releasable vesicles during recovery from short-term synaptic depression. Neuron 23:821-832.

Wu XS, McNeil BD, Xu J, Fan J, Xue L, Melicoff E, Adachi R, Bai L, Wu LG (2009) $\mathrm{Ca}(2+)$ and calmodulin initiate all forms of endocytosis during depolarization at a nerve terminal. Nat Neurosci 12:1003-1010.

Xu J, McNeil B, Wu W, Nees D, Bai L, Wu LG (2008) GTP-independent rapid and slow endocytosis at a central synapse. Nat Neurosci 11:45-53.

Yamashita T, Hige T, Takahashi T (2005) Vesicle endocytosis requires dynamin-dependent GTP hydrolysis at a fast CNS synapse. Science 307:124-127.

Yamashita T, Eguchi K, Saitoh N, von Gersdorff H, Takahashi T (2010) Developmental shift to a mechanism of synaptic vesicle endocytosis requiring nanodomain $\mathrm{Ca}^{2+}$. Nat Neurosci 13:838-844. 